\title{
CMB lensing reconstruction in the presence of diffuse polarized foregrounds
}

\section{Y. Fantaye*}

Institute of Theoretical Astrophysics, University of Oslo, P.O. Box 1029 Blindern, N-0315 Oslo, Norway

E-mail:y.t.fantayedastro.uio.no

\section{Baccigalupi}

SISSA, Astrophysics Sector, via Bonomea 265, Trieste 34136, Italy

INFN, Sezione di Trieste, Via Valerio 2, I-34151 Trieste, Italy

\section{S. M. Leach}

SISSA, Astrophysics Sector, via Bonomea 265, Trieste 34136, Italy

\section{A. P. S. Yadav \\ Department of Physics, University of California at San Diego, La Jolla, CA 92093}

\begin{abstract}
We perform a case study of a three-channel balloon-borne CMB experiment observing the sky at $(l, b)=\left(250^{\circ},-38^{\circ}\right)$ and attaining a sensitivity of $5.25 \mu \mathrm{K}$-arcmin with $8^{\prime}$ angular resolution at 150 $\mathrm{GHz}$, in order to assess whether the effect of polarized Galactic dust is expected to be a significant contaminant to the lensing signal reconstructed using the $E B$ quadratic estimator. We find that for our assumed dust model, polarization fractions of about as low as a few percent may lead to a significant dust bias to the lensing convergence power spectrum. For mitigating the effect of this dust bias we investigated a template cleaning method as well as removing foreground contaminated CMB modes from lensing estimation, and show that both methods are able to recover unbiased convergence power spectrum.
\end{abstract}

Big Bang, Big Data, Big Computers,

September 19-21, 2012

Laboratoire Astroparticule et Cosmologie, 10 rue A. Domon et L. Duquet, 75205 Paris 13, France

\footnotetext{
${ }^{*}$ Speaker.
} 


\section{Introduction}

The measurement and characterizaton of the weak lensing of the cosmic microwave background (CMB) by the large-scale structure distribution is a promising and active field of research in observational cosmology (for a review of the physics of CMB weak lensing, see [1]). Measurements of this signal can break fundamental degeneracies that afflict the cosmological interpretation of measurements of the CMB power spectrum [2] as well as help to improve the constraints on the cosmological parameters [3,4]. As a result, a number of ongoing and planned experiments are targeting the weak lensing signal as one of their primary science goals.

The Atacama Cosmology Telescope (ACT) collaboration made the first detection of weak lensing signal using CMB data alone [5] and the South Pole Telescope (SPT) collaboration have followed with a detection at higher significance [6] as well as detecting the correlation of the weak lensing 'convergence' and large-scale structure tracers from the Wide-field Infrared Survey Explorer and Spitzer/IRAC [7]. First applications of the weak lensing signal measurements have been to provide corroborating evidence for the cosmological constant from CMB data alone [8] and to improve constraints on the dark energy equation of state [6]. In the future, improved cosmological constraints are expected from the full SPT and ACT surveys, and especially from Planck which is poised to significantly advance lensing studies $[9,10]$.

Astrophysical foregrounds represent a major source of contamination to the lensing signal $[11,6,12,9]$. Specially this is true for lensing reconstruction from CMB polarization data, which is dominated by Galactic diffuse foregrounds. To date there has been no specific study on the possible impact of Galactic polarized dust emission on the detection of the lensing signal.

In the light of several ongoing ground-based and balloon-borne CMB polarization experiments including ACTPol [13], SPTPol [14], EBEX [15] and POLARBEAR [16], in this work we perform a case study of an EBEX-like experimental configuration.

\section{CMB and polarized dust simulations}

We simulate $\mathrm{CMB}$ polarization and diffuse polarized dust emission on a $13^{\circ} \times 13^{\circ}$ patch of sky located at $(\mathrm{RA}, \mathrm{Dec})=\left(75^{\circ},-44.5^{\circ}\right)$, corresponding to $(l, b)=\left(250^{\circ},-38^{\circ}\right)$ in Galactic coordinates. We assume a three band experimental configuration with channels at 150, 250 and 410 $\mathrm{GHz}$ observing to depths of 5.25, 14.0 and $140 \mu K_{\mathrm{CMB}}-$ arcmin respectively, each with an angular resolution of $8^{\prime}$.

The intensity of our Galactic polarized dust model is given by the model of [17] extrapolated to $410 \mathrm{GHz}$. Then, to simulate polarized emission, polarization angles are set on large angular scales using the WMAP dust template [18], while on smaller scales, extra Gaussian power is added using the prescription of [19]. The polarization fraction, $p$, is assumed to be spatially constant, and we investigate three cases of 3.6, 5, and 10\%, intended to bracket the average high Galactic latitude dust polarization detected in the WMAP W band [20], and possible higher dust polarization fractions observed by ARCHEOPS at $353 \mathrm{GHz}$ [21]. The dust is scaled from the $410 \mathrm{GHz}$ band to the lower frequency bands assuming a greybody frequency scaling with $T=18 \mathrm{~K}$ and $\beta=1.65$, with the dust temperature and spectral index both assumed to be uniform across the patch. 
For our CMB simulations, we produced two sets of 100 realizations-lensed and unlensedwith $0.76^{\prime}$ pixel size, assuming the WMAP 7-year best-fit cosmological parameter values [22], and with our fiducial CMB polarization power spectra calculated using CAMB [23].

The first set of maps-the lensed CMB realizations-were obtained starting from the unlensed fiducial power spectrum $C_{\ell}^{X Y}$, from which Gaussian realizations of the CMB polarization were generated, which were then lensed by remapping the pixels by the deflection field. The deflection field is in turn is derived from a Gaussian realization of the projected potential power spectrum

$C_{\ell}^{\phi \phi}$; we neglect the effect of the integrated Sachs-Wolfe effect induced correlation $C_{\ell}^{X \phi}$. We have chosen our pixel size to be small compared to the RMS of the deflection angles $\left(\sim 2^{\prime}\right)$ so that errors due to interpolation back onto the regular grid after remapping are small. We have checked that the $E$ and $B$-mode power spectra of these simulated lensed maps reproduces the lensed power spectra obtained from CAMB to within a few percent accuracy for the $E$-mode spectrum and to within five percent accuracy for the $B$-mode spectrum. While this is less accurate than the all-sky lensing simulations now performed by several groups using various interpolation schemes [24] [25] [26] [4], we believe that our flat-sky simulations are sufficiently accurate for our dust foreground study.

The second set of maps-the unlensed CMB realizations-were obtained from Gaussian realizations of the lensed fiducial power spectrum, $\tilde{C}_{\ell}^{X Y}$. These maps have the same power spectrum as the lensed CMB realizations, but have none of the lensing-induced non-Gaussianity. Since the power spectrum of the convergence reconstructed on unlensed CMB maps is same as the lensing noise power spectrum derived analytically, these maps have been used for checking the accuracy and implementation details of the convergence and power spectrum estimators, as well as the testing effect of mask apodization.

Finally the CMB maps are scaled to antenna temperature units in the three bands at 150, 250 and $410 \mathrm{GHz}$, smoothed with an $8^{\prime}$ beam, and uncorrelated Gaussian white noise is added to each pixel.

\section{Results}

This section describes our results in which we calculate the level of lensing bias that is expected from our dust polarization model.

Our convergence reconstruction pipeline, the $\mathrm{Hu}$ and Okamoto EB quadratic estimator [27], is validated under the most idealized foreground-free case. We used the CMB modes in the range $l_{\min }<\ell<l_{\max }$, where the minimum multipole is chosen to be twice the Nyquist mode, $k_{\text {nyq }}=$ $\pi / \Delta \theta$, where $\Delta \theta$ is the angular size of the patch in radians, while the maximum multipole is determined by the noise level and beam size of the experiment. For our case $l_{\min }=28$, and $l_{\max }=$ 3000. As we will show later, the choice of $l_{\min }$ becomes important when investigating the effect of foregrounds, while varying $l_{\max }$ does not significantly change our results.

Our pipline recovered unbiased estimates for two noise and beam cases we tested: 5.25 $\mu K$-arcmin sensitivity and $8^{\prime}$ angular resolution, EBEX type experiment, and a survey with $4 \mu K$-arcmin sensitivity and $1.4^{\prime}$ angular resolution similar to the planned 'ACTPol Deep' survey of [13]. The variance obtained from a 100 Monte Carlo simulation is in agreement with

$$
\Delta C_{\ell}^{\kappa \kappa}=\frac{\hat{C}_{\ell}^{\kappa \kappa}+N_{\ell}^{\kappa, X Y}}{\sqrt{\ell \Delta \ell f_{\text {sky }}}},
$$



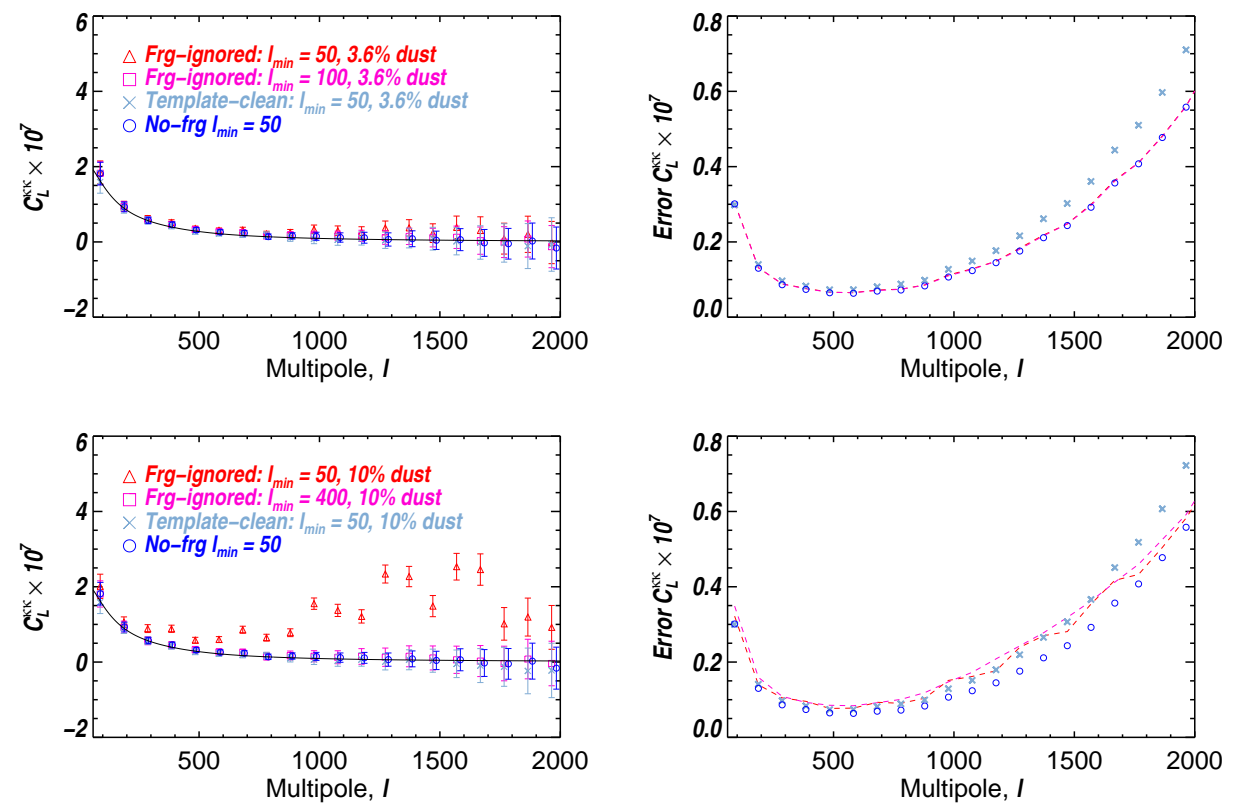

Figure 1: Template cleaning vs removing foreground contaminated modes for debiasing lens reconstruction. The left and right columns show the convergence power spectra and lensing noise ratios (relative to the foreground-free case) respectively, while the upper and lower rows are for the $p=0.1$ and $p=0.036$ cases respectively. Both methods effectively recovers unbiased lensing estimates for low and high polarization fractions at the minimal cost of lensing noise.

For these tests as well as for our study below we have applied a binning scheme $\Delta l=98$ (thirty bins between $\ell=40$ and $\ell=3000$ ).

\subsection{Dust polarization bias at $150 \mathrm{GHz}$}

To first assess the size of the dust contamination on the patch we are considering, we estimated the power spectrum of the simulated dust at $150 \mathrm{GHz}$ and compared it to $E$ and $B$-mode signal and noise power spectra. For our dust model and choice of patch, the $E$ and $B$-mode power spectra of the dust approximately follow a powerlaw given by $C_{\ell}^{\text {dust }}=(A \times p)^{2} \ell^{\beta}$, where $p$ is the polarization fraction, $A \simeq 120 \mu \mathrm{K}$ and $\beta \simeq-3.5$. Our previous study [28] has shown that polarized dust at this level of power must be modeled and subtracted in order to derive unbiased estimated of the inflationary $B$-mode spectrum, a cosmological signal which is accessible in the $\ell<200$ range of the $B$-mode power spectrum. The main question we seek to address in this study is whether this level of anisotropy power of foreground contamination is large enough to also bias the estimates of the lensing signal.

We have calculated the power spectrum of the convergence field reconstructed with the $E B$ quadratic estimator using the dust contaminated $150 \mathrm{GHz}$ channel, and show our results in Figure 1 for two different polarization fractions of $p=[0.036,0.1]$. We find that if the dust contamination is ignored during the lensing estimation, then a 'dust noise bias' dominates over the lensing power spectrum estimates for $p=0.1$, and slightly biases the $p=0.036$. 
Although our demonstration of the dust bias will be dependent on the choice of patch we have assumed, and on the details of our polarized dust model and its power spectrum, we nonetheless conclude that diffuse polarized dust may in principle be a source of bias for future sub-orbital $\mathrm{CMB}$ surveys aiming at lensing estimates using the $E B$ estimator, and that methods for foreground debiasing must therefore be developed.

\subsection{Discussion}

To mitigate the impact of lensing bias due to polarized dust, firstly we considered whether template-based foreground cleaning methods [29, 30, 31] can allow unbiased estimate of the convergence power spectrum. We used an approximate template-based cleaning method in which the dust-dominated $410 \mathrm{GHz}$ channel is used as a polarized dust template to suppress the foreground contamination in the $150 \mathrm{GHz}$ channel. The dust amplitude coefficient, $\alpha_{d}$, is estimated by maximising the likelihood

$$
-2 \ln \mathscr{L}=\sum_{p} \frac{\left(Q_{150}-\alpha_{d} \times Q_{410}\right)^{2}}{\sigma_{Q, 150}^{2}}+\frac{\left(U_{150}-\alpha_{d} \times U_{410}\right)^{2}}{\sigma_{U, 150}^{2}},
$$

where the pixel size has first been degraded to $6.1^{\prime}$. Once the template coefficient has been estimated then the full resolution maps are appropriately combined, and the noise is propagated using

$$
\left[\sigma_{Q}^{2}, \sigma_{U}^{2}\right]=\frac{\left[\sigma_{Q}^{2}, \sigma_{U}^{2}\right]^{150}+\alpha_{d}^{2}\left[\sigma_{Q}^{2}, \sigma_{U}^{2}\right]^{410}}{\left(1-\alpha_{d}\right)^{2}}
$$

Figure ?? shows our results from our template cleaning as well as low multipole filtering we will discuss below. Our basic finding is that at lease for the foreground model we considered, with a constant dust spectral index scaling, template cleaning provides a robust alternative for unbiased reconstruction of the lensing field, in the sense that the final noise level of the cleaned CMB estimate is fairly insensitive to the level of foreground contamination.

Secondly, since the dust contamination has a 'red' anisotropy power spectrum, and this suggests a possible strategy for mitigating the dust bias. As long as we have information about the power spectrum of dust, then filtering the low-multipole modes can be used to reduce the bias $[32,6]$, perhaps at an acceptable cost to the variance. We have demonstrated this technique by varying $l_{\min }$, and found that appropriate tuning of this parameter can indeed reduce the dust bias effect. Specifically we found that the value of $l_{\min }$ that results in unbiased estimates of the convergence power spectrum depends on the polarization fraction of the foreground: the greater the foreground level, the more aggressive the required low-multipole filtering. For our dust model and choice of patch, the approximate required filtering scale is given by $l_{\min } \sim 100 \times(p / 0.036)$. For dust polarization fractions less than 0.05 , we found that the loss in the signal due to the $l_{\min }$ cut is small enough to yield error bars close to the foreground-free case. In Figure 1, we show the performance of low multipole filtering. We used $\ell_{\min }=400$ for $p=0.1$ and $\ell_{\min }=100$ for $p=0.036$. In both cases the input convergence power spectrum is recovered with only a slight increase in the variance with respect to a no foreground case. 


\section{Conclusions}

Several ongoing and planned CMB polarization experiments are aiming to measure and characterise the lensing of the cosmic microwave background, in order to improve constraints on the parameters of the cosmological model. Within this context we have made the first specific study of the possible effect of diffuse polarized dust emission on the accuracy of the reconstruction of the lensing convergence signal. Our particular focus has been on performing a case study of a three channel balloon-borne CMB experiment covering the frequency range 150-410 GHz. Our numerical investigation is based on a dust polarization simulation and a flat-sky implementation of the $\mathrm{Hu}$ and Okamoto quadratic estimator [27]. We found that for the sky patch under consideration, which is near to the region of sky that will be targeted by the EBEX experiment, and for plausible dust polarization fractions in the range 3.6-10\%, the anisotropy of the diffuse dust polarization will be large enough at $150 \mathrm{GHz}$ to bias the reconstruction of the convergence. Thus a multi-frequency experimental approach is imperative, and appropriate analysis methods must be developed for debiasing the effect of polarized dust.

In order to mitigate the effect of the dust and to debias the convergence power spectrum, we demonstrated that removing the lower-multipole foreground-contaminated CMB modes from the

lensing reconstruction, as well as using the $410 \mathrm{GHz}$ channel as a dust template provide two further methods for diffuse foreground mitigation.

These results were first presented in [33]

\section{Acknowledgments}

YF would like to thank the organizers of the BIG3 workshop, and SISSA for funding the expenses to attend the workshop.

\section{References}

[1] A. Lewis and A. Challinor. Weak gravitational lensing of the CMB. Phys. Rep., 429:1-65, June 2006.

[2] R. Stompor and G. Efstathiou. Gravitational lensing of cosmic microwave background anisotropies and cosmological parameter estimation. MNRAS, 302:735-747, February 1999.

[3] L. Perotto, et al. Probing cosmological parameters with the CMB: forecasts from Monte Carlo simulations. Journal of Cosmology and Astro-Particle Physics, 10:13-+, October 2006.

[4] A. Benoit-Lévy, K. M. Smith, and W. Hu. Non-Gaussian structure of the lensed CMB power spectra covariance matrix. ArXiv e-prints, May 2012.

[5] S. Das, et al. Detection of the Power Spectrum of Cosmic Microwave Background Lensing by the Atacama Cosmology Telescope. Physical Review Letters, 107(2):021301, July 2011.

[6] A. van Engelen, et al. A measurement of gravitational lensing of the microwave background using South Pole Telescope data. ArXiv e-prints, February 2012.

[7] L. E. Bleem, et al. A Measurement of the Correlation of Galaxy Surveys with CMB Lensing Convergence Maps from the South Pole Telescope. ArXiv e-prints, March 2012. 
[8] B. D. Sherwin, et al. Evidence for Dark Energy from the Cosmic Microwave Background Alone Using the Atacama Cosmology Telescope Lensing Measurements. Physical Review Letters, 107(2):021302, July 2011.

[9] L. Perotto, et al. Reconstruction of the cosmic microwave background lensing for Planck. A\&A, 519:A4, September 2010.

[10] D. Hanson, et al. CMB temperature lensing power reconstruction. Phys. Rev. D., 83(4):043005, February 2011.

[11] A. Amblard, C. Vale, and M. White. Weak lensing of the CMB by large-scale structure. New A, 9:687-704, October 2004.

[12] K. M. Smith, et al. Gravitational Lensing. In S. Dodelson, D. Baumann, A. Cooray, J. Dunkley, A. Fraisse, M. G. Jackson, A. Kogut, L. Krauss, M. Zaldarriaga, \& K. Smith, editor, American Institute of Physics Conference Series, volume 1141 of American Institute of Physics Conference Series, pages 121-178, June 2009.

[13] M. D. Niemack, et al. ACTPol: a polarization-sensitive receiver for the Atacama Cosmology Telescope. In Society of Photo-Optical Instrumentation Engineers (SPIE) Conference Series, volume 7741 of Society of Photo-Optical Instrumentation Engineers (SPIE) Conference Series, July 2010.

[14] J. J. McMahon, et al. SPTpol: an instrument for CMB polarization. In B. Young, B. Cabrera, \& A. Miller, editor, American Institute of Physics Conference Series, volume 1185 of American Institute of Physics Conference Series, pages 511-514, December 2009.

[15] B. Reichborn-Kjennerud, et al. EBEX: a balloon-borne CMB polarization experiment. In Society of Photo-Optical Instrumentation Engineers (SPIE) Conference Series, volume 7741, July 2010.

[16] A. T. Lee, et al. POLARBEAR: Ultra-high Energy Physics with Measurements of CMB Polarization. In H. Kodama \& K. Ioka, editor, American Institute of Physics Conference Series, volume 1040 of American Institute of Physics Conference Series, pages 66-77, August 2008.

[17] D. J. Schlegel, D. P. Finkbeiner, and M. Davis. Maps of Dust Infrared Emission for Use in Estimation of Reddening and Cosmic Microwave Background Radiation Foregrounds. ApJ, 500:525-+, June 1998.

[18] L. Page et al. Three year Wilkinson Microwave Anisotropy Probe (WMAP) observations: Polarization analysis. Astrophys. J. Suppl., 170:335, 2007.

[19] G. Giardino, et al. Towards a model of full-sky Galactic synchrotron intensity and linear polarisation: A re-analysis of the Parkes data. A\&A, 387:82-97, May 2002.

[20] A. Kogut, et al. Three-Year Wilkinson Microwave Anisotropy Probe (WMAP) Observations: Foreground Polarization. ApJ, 665:355-362, August 2007.

[21] A. Benoit, et al. First detection of polarization of the submillimetre diffuse galactic dust emission by Archeops. A\&A, 424:571-582, September 2004.

[22] E. Komatsu, et al. Seven-year Wilkinson Microwave Anisotropy Probe (WMAP) Observations: Cosmological Interpretation. ApJS, 192:18-+, February 2011.

[23] A. Lewis, A. Challinor, and A. Lasenby. Efficient Computation of Cosmic Microwave Background Anisotropies in Closed Friedmann-Robertson-Walker Models. ApJ, 538:473-476, August 2000.

[24] A. Lewis. Lensed CMB simulation and parameter estimation. Phys. Rev. D., 71(8):083008-+, April 2005. 
[25] S. Basak, S. Prunet, and K. Benabed. Simulating weak lensing of CMB maps. A\&A, 508:53-62, December 2009.

[26] G. Lavaux and B. D. Wandelt. Fast and Optimal Cosmic Microwave Background Lensing Using Statistical Interpolation on the Sphere. ApJS, 191:32-42, November 2010.

[27] W. Hu and T. Okamoto. Mass Reconstruction with Cosmic Microwave Background Polarization. ApJ, 574:566-574, August 2002.

[28] Y. Fantaye, et al. Estimating the tensor-to-scalar ratio and the effect of residual foreground contamination. J. Cosmology Astropart. Phys., 8:1, August 2011.

[29] L. Page, et al. Three-Year Wilkinson Microwave Anisotropy Probe (WMAP) Observations: Polarization Analysis. ApJS, 170:335-376, June 2007.

[30] G. Efstathiou, S. Gratton, and F. Paci. Impact of Galactic polarized emission on B-mode detection at low multipoles. MNRAS, 397:1355-1373, August 2009.

[31] N. Katayama and E. Komatsu. Simple Foreground Cleaning Algorithm for Detecting Primordial B-mode Polarization of the Cosmic Microwave Background. ApJ, 737:78, August 2011.

[32] W. Hu, S. DeDeo, and C. Vale. Cluster mass estimators from CMB temperature and polarization lensing. New Journal of Physics, 9:441, December 2007.

[33] Y. Fantaye, et al. CMB lensing reconstruction in the presence of diffuse polarized foregrounds. $J$. Cosmology Astropart. Phys., 12:17, December 2012. 